\title{
Dynamic changes of $\alpha$-melanocyte-stimulating hormone levels in the serum of patients with craniocerebral trauma
}

\author{
XIAOGUANG DU, BAOZHONG DONG, CHEN LI, FAXUE ZHANG, \\ YANWEI JI, JIANBIN ZHANG and CHANGJIANG YIN
}

Department of Neurosurgery, Shandong Jiaotong Hospital, Jinan, Shandong 250031, P.R. China

Received March 17, 2017; Accepted July 4, 2017

DOI: $10.3892 / \mathrm{etm} .2017 .4793$

\begin{abstract}
The aim of the study was to investigate dynamic changes in $\alpha$-melanocyte-stimulating hormone $(\alpha-\mathrm{MSH})$ levels in the serum of patients with craniocerebral trauma. Forty-eight patients with acute craniocerebral injury were selected between January 2015 and October 2016. The patients were divided into three groups: severe (18 cases), moderate (16 cases) and mild (14 cases), according to the Glasgow Coma Scale (GCS) score at the time of admission. At the same time, 10 adults with a similar age distribution to the patients were also selected as a control group. Venous blood was extracted from patients at 1, 3, 5 and 7 days after injury. Serum $\alpha-\mathrm{MSH}$ and tumor necrosis factor (TNF)- $\alpha$ levels were measured using an enzyme-linked immunosorbent assay (ELISA). The correlation between $\alpha$-MSH and TNF- $\alpha$ was analyzed using Pearson's correlation analysis. Serum $\alpha$-MSH levels in patients with craniocerebral injury were lower than those in the healthy control group $(\mathrm{P}<0.05)$. Decreased serum $\alpha$-MSH levels were usually accompanied with higher degrees of craniocerebral injury. Serum $\alpha$-MSH levels initially decreased and then later increased, with the lowest $\alpha$-MSH levels in the mild at 5 days, moderate at 5 days, and severe groups at 3 days after injury $(\mathrm{P}<0.05)$. Serum TNF- $\alpha$ levels in all the patient groups were higher than those in the control group at different time points after injury, with higher TNF- $\alpha$ serum levels accompanying higher degrees of brain injury. In all three groups, serum TNF- $\alpha$ levels initially increased and then decreased post-injury, with peak serum TNF- $\alpha$ levels found at 3-day postinjury in all the patient groups $(\mathrm{P}<0.05)$. A negative correlation between serum $\alpha$-MSH content and serum TNF- $\alpha$ levels in patients with craniocerebral trauma at different time points, was noted $(\mathrm{P}<0.05)$. Serum $\alpha-\mathrm{MSH}$ content in the survival
\end{abstract}

Correspondence to: Dr Baozhong Dong, Department of Neurosurgery, Shandong Jiaotong Hospital, 12 Wuyingshan Middle Road, Jinan, Shandong 250031, P.R. China

E-mail: dxg05n@163.com

Key words: $\alpha$-melanocyte-stimulating hormone craniocerebral trauma, inflammatory factors, systemic inflammatory response syndrome, tumor necrosis factor- $\alpha$ group was higher than that in the death group $(\mathrm{P}<0.05)$. Serum $\alpha$-MSH levels in patients with non-systemic inflammatory response syndrome (SIRS) were higher than in patients with SIRS $(\mathrm{P}<0.05)$. Serum $\alpha$-MSH levels during the early stages after craniocerebral trauma can be used as a factor for the prediction of secondary SIRS, with constant low levels of serum $\alpha$-MSH suggest poor prognosis.

\section{Introduction}

Craniocerebral trauma is attracting increasing attention due to treatment difficulty, high costs and high morbidity and mortality rates. At present, the incidence of craniocerebral trauma is high throughout the world and has become the primary cause of disability and death of young adults in developed countries $(1,2)$. An epidemiological survey in China showed that the disability rate was $60 \%$ in patients with moderate craniocerebral injury and $100 \%$ in patients with severe craniocerebral injury (3).

Craniocerebral trauma, not only causes direct external injury, but also induces inflammation-mediated secondary injury (4). Local leukocyte accumulation and activation after craniocerebral trauma can cause the release of large amounts of inflammatory factors such as interleukin (IL), tumor necrosis factor- $\alpha$ (TNF- $\alpha$ ) and nitric oxide (NO). These inflammatory factors induce and mediate a series of pathological changes including increased cerebral vascular permeability, brain edema, intracranial hypertension and brain tissue irrigation, resulting in brain cell death (5). Activated neutrophils and macrophages release large amounts of proteases and oxygen-free radicals after craniocerebral trauma, which not only aggravates brain cell damage, but also damages the cerebral vasculature and causes bleeding, so that the original hematoma in brain promotes the induction of delayed hematoma (6). The accumulation of inflammatory factors after craniocerebral trauma can cause systemic inflammatory response syndrome (SIRS), with severe SIRS eventually leading to the occurrence of acute respiratory distress syndrome and multiple organ failure (7). Controlling craniocerebral trauma after this inflammatory reaction can effectively prevent craniocerebral edema, reduce trauma and reduce the possibility of re-bleeding after surgical treatment $(4,5)$. $\alpha$-Melanocyte-stimulating hormone ( $\alpha$-MSH), an endogenous peptide secreted by the body, is derived from 
$\alpha$-melanocortin (POMC). $\alpha$-MSH plays a role in various physiological processes (8). Recent findings have shown that $\alpha$-MSH exerts antibacterial functions and participates in the regulation of obesity and sexual function. $\alpha$-MSH was also found to play a protective role in nervous system injury, mainly through its anti-inflammatory effects $(9,10), \alpha-\mathrm{MSH}-$ mediated mechanisms in anti-inflammatory processes include: inhibition of $\mathrm{NF}-\kappa \mathrm{B}$ gene activation to reduce inflammatory factor production at the genetic level, direct binding to glial cells of the CNS and peripheral neutrophils to inhibit the inflammatory response (11) and inhibition of peripheral inflammatory responses through central nervous system signaling pathways (12). The application of $\alpha-\mathrm{MSH}$ is safe and the duration of the effect is long. $\alpha$-MSH can pass through the blood-brain barrier, providing a novel new option for clinical treatment $(6,7)$.

At present, few studies exist regarding serum $\alpha$-MSH levels. Previous studies showed that synovitis leads to increased $\alpha$-MSH content in intra-articular fluid in normal individuals, but serum $\alpha$-MSH content did not increase $(13,14)$. As an endogenous anti-inflammatory peptide, serum changes in $\alpha$-MSH levels after craniocerebral trauma are worthy of study. In the present study, patients were divided into mild, moderate, and severe groups using the Glasgow Coma Scale (GCS) score at the time of admission. Serum $\alpha-\mathrm{MSH}$ and TNF- $\alpha$ levels in patients with craniocerebral trauma were measured by double antibody sandwich enzyme-linked immunosorbent assay (ELISA) at multiple time points to investigate changes in serum $\alpha$-MSH content after craniocerebral trauma and its relationship with TNF- $\alpha$. The correlation between $\alpha-\mathrm{MSH}$ and the severity and prognosis of SIRS was also investigated.

\section{Materials and methods}

Clinical data. Forty-eight patients with acute craniocerebral trauma were selected between January 2015 and October 2016 in the emergency department of Shandong Jiaotong Hospital (Shandong, China) to serve as the observation group. Of the selected patients, 38 were male and 10 female, with age ranging from 16 to 72 years (average, 43.1 years of age). All the patients were treated at Shandong Jiaotong Hospital within $24 \mathrm{~h}$ after injury. Of the 48 patients, brain contusion and laceration were found in 16 cases, intracranial hematoma was found in 12 cases, subdural hematoma or subdural hematoma combined with epidural hematoma was found in 8 cases, diffuse axonal injury was found in 6 cases and complex craniocerebral injury was found in 6 cases. Patient selection criteria were as follows: CT, MRI and other imaging tests were used to confirm diagnosis. Patients with injury in other organs were excluded. Patients were required to have no history of neurological damage, no chronic organ injury and blood diseases, no cancer and other wasting diseases and no history of infection before the injury. An informed consent form was signed by the patients or their family members. Patients were divided into mild (12-15 points; 14 cases), moderate (8-12 points; 16 cases) and severe (3-8 points; 18 cases) groups using the GCS scoring criteria. Statistical analysis found no differences in age and gender between groups. The control group included 10 normal adults selected based on physical examinations at
Shandong Jiaotong Hospital. This study was approved by the Ethics Committee of Shandong Jiaotong Hospital. Signed written informed consents were obtained from the patients and/or guardians.

Specimen collection and storage. Basic patient information was recorded and routine clinical monitoring was performed for all patients. Venous blood was extracted from patients at $1,3,5$, and 7 days after injury. Fasting venous blood was extracted once from the healthy adults in the control group. Blood was placed into blood collection tubes and stored at room temperature for $20 \mathrm{~min}$. After blood coagulation, the sample was centrifuged for $15 \mathrm{~min}$ at $2,650 \mathrm{x} \mathrm{g}$. The supernatant (serum) was collected and injected into sealed tubes and stored in an ultra-low refrigerator at $-70^{\circ} \mathrm{C}$.

Experimental methods, materials, and instrument. Experimental materials and equipment. $\alpha-\mathrm{MSH}$ and TNF- $\alpha$ ELISA kits were purchased from Wuhan Boster Biological Engineering Co. Ltd. (Hubei, China). The following equipment was used in the present study: microplate reader, whirlpool mixer (Shanghai Yuejin Medical Equipment Co. Ltd., Shanghai, China); $-70^{\circ} \mathrm{C}$ ultra-low temperature refrigerator (Sony, Japan); cylinders with different ranges (Beijing Zhongyi Hongrui Science \& Technology Development Co. Ltd.); CL5R high-speed centrifugal machine (Thmorgan Biotechnology Co. Ltd., Beijing, China).

Experimental methods. Mouse monoclonal anti-human $\alpha$-MSH antibody (dilution, 1:100; cat. no. 187692) and mouse monoclonal anti-human TNF- $\alpha$ monoclonal antibody (dilution, 1;100; cat. no. 189846), purchased from Wuhan Sanying Biotech (Wuhan, Hubei, China) were coated on ELISA plates. After $\alpha$-MSH and TNF- $\alpha$ in standard or experimental samples had bound the monoclonal antibody, the ELISA plate was washed. Following this, biotinylated anti-human $\alpha-\mathrm{MSH}$ and anti-human TNF- $\alpha$ secondary antibodies were added to form immune complexes. After washing, horseradish peroxidaselabeled streptavidin was added to bind biotin. After washing again, the substrate was added and the reaction made the solution turn blue. A sulfuric acid solution was then added to stop the reaction. The optical density (OD) value of the solution in each well of the enzyme plate was measured at a wavelength of $450 \mathrm{~nm}$ using a microplate reader within $15 \mathrm{~min}$. The concentrations of $\alpha$-MSH and TNF- $\alpha$ were proportional to the OD value. According to the known concentrations of the standard samples and the measured OD value, the standard curve was drawn to calculate the concentrations of $\alpha-\mathrm{MSH}$ and TNF- $\alpha$ in experimental samples.

Statistical analysis. Experimental data were analyzed using SPSS 17.0 software (IBM, Armonk, NY, USA). Measurement data were expressed as mean \pm standard deviation. Repeated measures analysis of variance and one-way ANOVA were applied. The LSD-T method was used for comparisons of data between more than 2 groups. Comparisons of data between 2 groups were performed using the t-test. $\mathrm{P}<0.05$ was considered to indicate a statistically significant difference. Pearson's correlation analysis was used to analyze the correlation between the two indicators. 
Table I. Serum $\alpha$-MSH content in trauma and control groups at each time point $(\mathrm{pg} / \mathrm{ml})$.

\begin{tabular}{|c|c|c|c|c|c|c|c|}
\hline Groups & $\mathrm{n}$ & 1 day & 3 days & 5 days & 7 days & F-value & P-value \\
\hline Control & 10 & $45.674 \pm 10.952$ & $45.674 \pm 10.952$ & $45.674 \pm 10.952$ & $45.674 \pm 10.952$ & 0.527 & 0.483 \\
\hline Mild & 14 & $30.204 \pm 7.077^{\mathrm{a}, \mathrm{d}}$ & $23.639 \pm 5.154^{\mathrm{a}, \mathrm{d}}$ & $18.886 \pm 7.186^{\mathrm{a}}$ & $23.326 \pm 7.875^{\mathrm{add}}$ & 1.278 & 0.011 \\
\hline Moderate & 16 & $17.318 \pm 4.571^{\mathrm{a}, \mathrm{b}, \mathrm{d}}$ & $13.110 \pm 4.542^{\mathrm{a}, \mathrm{b}}$ & $10.692 \pm 0.0430^{\mathrm{a}, \mathrm{b}}$ & $23.827 \pm 22.831^{\mathrm{a}, \mathrm{d}}$ & 1.478 & 0.008 \\
\hline Severe & 18 & $11.594 \pm 3.508^{\mathrm{a}-\mathrm{c}, \mathrm{e}}$ & $9.649 \pm 4.205^{\mathrm{a}-\mathrm{c}}$ & $11.647 \pm 5.354^{\mathrm{a}, \mathrm{b}, \mathrm{e}}$ & $19.022 \pm 14.595^{\mathrm{ae}}$ & 1.272 & 0.012 \\
\hline
\end{tabular}

One-way ANOVA, $\mathrm{P}<0.05$ vs. ${ }^{\mathrm{a}}$ control, ${ }^{\mathrm{b}}$ mild and ${ }^{\mathrm{c}}$ moderate groups; Student's t-test, $\mathrm{P}<0.05$ vs. 5 days in ${ }^{\mathrm{d}}$ mild and moderate and 5 days in esevere group.

Table II. Serum TNF- $\alpha$ content in the trauma and control groups at each time point (pg/ml).

\begin{tabular}{|c|c|c|c|c|c|}
\hline Groups & No. & 1 day & 3 days & 5 days & 7 days \\
\hline Control & 14 & $12.430 \pm 2.865^{\mathrm{a}}$ & $18.597 \pm 7.825^{\mathrm{a}}$ & $14.441 \pm 3.525^{\mathrm{a}}$ & $13.829 \pm 7.653^{\mathrm{a}}$ \\
\hline Moderate & 16 & $20.685 \pm 5.990^{\mathrm{a}, \mathrm{b}, \mathrm{d}, \mathrm{e}}$ & $26.188 \pm 6.780^{\mathrm{a}, \mathrm{b}}$ & $20.114 \pm 2.389^{\mathrm{a}, \mathrm{b}, \mathrm{d}, \mathrm{e}}$ & $15.752 \pm 2.544^{\mathrm{a}, \mathrm{b}, \mathrm{d}}$ \\
\hline Severe & 18 & $19.791 \pm 7.558^{\mathrm{a}, \mathrm{b}}$ & $37.239 \pm 18.280^{\mathrm{a}-\mathrm{c}}$ & $27.978 \pm 14.201^{\mathrm{a}-\mathrm{d}, \mathrm{f}}$ & $25.344 \pm 7.952^{\mathrm{a}-\mathrm{d}, \mathrm{f}}$ \\
\hline
\end{tabular}

One-way ANOVA, $\mathrm{P}<0.05$ vs. the ${ }^{\mathrm{a}}$ control, ${ }^{\mathrm{b}}$ mild and ${ }^{\mathrm{c}}$ moderate; $\mathrm{P}<0.05$ vs. 3 days in ${ }^{\mathrm{d}}$ each group; $\mathrm{P}<0.05$ vs. 7 days in ${ }^{\mathrm{e}}$ moderate group; $\mathrm{P}<0.05$ vs. with 1 day in ${ }^{\mathrm{f}}$ severe group.

\section{Results}

Comparison of serum $\alpha$-MSH levels in the trauma and control groups at different time points after injury. Statistically significant differences were found in serum $\alpha$-MSH levels between the trauma and control groups $(\mathrm{P}<0.05)$. Further comparisons between the different groups at each time point were performed. $\alpha$-MSH levels in the trauma groups were lower than those in the control group at each time point $(\mathrm{P}<0.05)$. Different trauma groups at 1 and 3 days after injury showed that lower serum $\alpha$-MSH levels were accompanied by higher levels of craniocerebral injury. Serum $\alpha$-MSH levels in the mild and severe groups were significantly lower than those in the mild group at 5 days after injury $(\mathrm{P}<0.05)$, but there was no significant difference between the moderate and severe groups. There was no significant difference in serum $\alpha$-MSH level between the different trauma groups at 7 days after injury $(\mathrm{P}>0.05)$. Statistically significant differences were found in serum $\alpha$-MSH levels in the same group at different time points after craniocerebral trauma. Multiple comparative analysis within each group showed that $\alpha$-MSH levels in the mild group at 1 day after injury were higher than those at 3,5 and 7 days after injury $(\mathrm{P}<0.05)$. There was a significant difference between the severe group serum $\alpha$-MSH levels at 3 and 5 days and levels at 7 days after injury $(\mathrm{P}<0.05)$. Serum $\alpha$-MSH levels were lowest in the mild group at 5 days after injury and the differences between the serum $\alpha$-MSH level at 5 days and serum $\alpha-\mathrm{MSH}$ level at 1,3 and 7 days were significant $(\mathrm{P}<0.05)$. Serum $\alpha-\mathrm{MSH}$ content in the moderate group was initially decreased and later increased, with serum $\alpha$-MSH levels at 5 days significantly different from those at 1 and 7 days $(\mathrm{P}<0.05)$. However, no significant difference was found between levels at 3 and 5 days.
Serum $\alpha$-MSH levels in the severe group were lowest at 3 days and levels at 3 days were significantly different from levels at other time points $(\mathrm{P}<0.05$, Table I).

Comparison of serum TNF- $\alpha$ levels in the trauma and control groups at different time points after injury. Statistically significant differences were found in serum TNF- $\alpha$ levels between the different groups $(\mathrm{P}<0.05)$. Further comparisons between the different groups at the same time point were performed. Serum TNF- $\alpha$ levels in the trauma groups were higher than those in the control group $(\mathrm{P}<0.05)$. Higher TNF- $\alpha$ levels were accompanied by a higher degree of injury at all time points $(\mathrm{P}<0.05)$. A significant difference was found in the serum TNF- $\alpha$ level between the trauma groups at all the time points $(\mathrm{P}<0.05)$. However, no significant difference was found between the moderate and severe groups at 1 day. There was a significant difference between the different time points in the same group $(\mathrm{P}<0.05)$. Multiple comparison within groups showed that serum TNF- $\alpha$ levels in all the trauma groups peaked at 3 days and this peak was significantly higher than TNF- $\alpha$ levels at other time points $(\mathrm{P}<0.05)$. Serum TNF- $\alpha$ levels in the three groups decreased after 3 days. In the moderate group, although serum TNF- $\alpha$ levels were still high at 5 and 7 days, they were significantly lower than at 3 days $(\mathrm{P}<0.05)$. Furthermore, serum TNF- $\alpha$ levels at 1 and 5 days were significantly different from 7 days $(\mathrm{P}<0.05)$. The serum TNF- $\alpha$ levels in the severe group peaked at 3 days and decreased slowly after that, with serum TNF- $\alpha$ levels at 5 and 7 days being still significantly higher than at 1 day $(\mathrm{P}<0.05$, Table II).

Correlation between $\alpha-M S H$ content and TNF- $\alpha$ content in serum after injury. There was a negative correlation between 
Table III. Correlation between serum $\alpha$-MSH content and TNF- $\alpha$ content after injury.

\begin{tabular}{lrccr}
\hline $\mathrm{n}=48$ & 1 day & 3 days & 5 days & 7 days \\
\hline Correlation & Negative & Negative & Negative & Negative \\
P-value & 0.011 & 0.005 & 0.02 & 0.009 \\
Correlation & -0.386 & -0.45 & -0.396 & -0.407 \\
coefficient & & & & \\
\hline
\end{tabular}

serum $\alpha$-MSH content and TNF- $\alpha$ level at 1 day $(r=-0.386)$, 3 days $(r=-0.45), 5$ days $(r=-0.396)$ and 7 days $(r=-0.407)$ after injury (Table III).

Comparison of serum $\alpha$-MSH levels between survival and death groups, and between SIRS and non-SIRS groups after craniocerebral trauma. Serum $\alpha$-MSH levels in the survival group were higher than those in the death group (Table IV, $\mathrm{P}<0.05$ ). Serum $\alpha$-MSH levels in the SIRS group at 1 day after injury were significantly lower than in the nonSIRS group at the same time point $(\mathrm{P}<0.05$, Table $\mathrm{V})$.

\section{Discussion}

Craniocerebral trauma is a worldwide public health problem with high incidence. Approximately 57 million individuals worldwide have been hospitalized due to craniocerebral trauma annually (9). A survey in United States showed that there were approximately 1.4 million new cases of craniocerebral trauma annually, causing different degrees of physical and mental disability in approximately 5.3 million individuals (9). Therefore, seeking new treatments and improving functional rehabilitation remains an important public issue.

Previous findings based on the inflammatory response caused by craniocerebral trauma presented a novel way to treat craniocerebral trauma by controlling inflammation. Apart from the direct primary injury, craniocerebral trauma can also cause inflammatory factor-mediated secondary injury. Reducing secondary injury after craniocerebral trauma plays a key role in reducing disability and mortality and improving prognosis (11-14). Experimental studies have shown that the inflammatory response is an important part of the pathophysiological processes underlying craniocerebral trauma, where the release of inflammatory factors is an important cause of secondary head injury (11). After craniocerebral trauma, immune cell activation generates chemokines and regulatory factors, allowing the activated immune cells to move towards the injured site and aggregate locally $(12,13)$. Immune cells facilitate the release of large amounts of $\mathrm{TNF}-\alpha$, resulting in increased brain tissue damage (13). The production of histamine, prostaglandins and other factors during this inflammatory reaction can increase brain vascular permeability, increase brain tissue edema and reduce brain tissue perfusion pressure (14). In addition, excessive inflammation of the brain after traumatic brain injury can also lead to the occurrence of SIRS, acute respiratory distress syndrome and multiple organ dysfunction syndromes, which in turn affect prognosis $(15,16)$. It has been confirmed that neuropeptides and neuroendocrine hormones are closely associated with systemic inflammatory responses. As an endogenous neuropeptide, changes in $\alpha-\mathrm{MSH}$ levels after certain injuries, as well as its potential application in clinical application, has attracted attention. Previous findings have confirmed the existence of large amounts of inflammatory factors in serum, cerebrospinal fluid and even brain tissue after craniocerebral trauma, with the amount associated with the degree of trauma. Persistent inflammation of the brain tissue increases the risk of development of mental illness (11-13).

In view of the clinical complications and poor prognosis caused by the post-craniocerebral trauma inflammatory response, the application of anti-inflammatory drugs in the clinical treatment of craniocerebral trauma has been investigated. Although steroidal drugs such as indomethacin can reduce brain edema, they cannot pass through the blood-brain barrier, limiting their clinical application (14). As an endogenous peptide, $\alpha$-MSH is mainly distributed in the thalamus, pituitary, spinal cord, gastrointestinal tract and other tissues (16). $\alpha$-MSH has a wide range of physiological effects exerted by binding to its receptors, including M1R-M5R, which are widely distributed in nervous system tissues (17). $\alpha$-MSH was first recognized because it can promote the deposition of melanin in the skin and its unique anti-inflammatory effects have garnered more attention with subsequent studies. $\mathrm{NF}-\kappa \mathrm{B}$ plays an important role in the regulation of the inflammatory response. $\alpha$-MSH can inhibit NF- $\kappa$ B gene expression by increasing the concentration of second messenger cAMP to prevent the degradation of I $\kappa$ B protein (18). $\alpha$-MSH receptors can be found on the surface of peripheral immune and glial cells with immune effects within the central nervous system, $\alpha$-MSH can directly affect immune cells by interacting with its receptors (18). In addition, $\alpha$-MSH can act on macrophages to inhibit inducible NO synthase (iNOS), thereby inhibiting the production of NO and neopterin, which in turn inhibits macrophage activation (18). In

Table IV. Serum $\alpha$-MSH levels in survival and death groups (pg/ml).

\begin{tabular}{lccccc}
\hline Variable & No. & 1 day & 3 days & 5 days & 7 days \\
\hline Survival group & 40 & $20.258 \pm 8.947$ & $15.813 \pm 7.252$ & $14.076 \pm 6.657$ & $24.604 \pm 16.846$ \\
Death group & 8 & $11.552 \pm 5.590$ & $9.556 \pm 5.346$ & $9.415 \pm 4.113$ & $8.060 \pm 2.885$ \\
t-test & - & 2.873 & 4.372 & 4.983 & 5.487 \\
P-value & - & 0.021 & 0.011 & 0.017 & 0.008 \\
\hline
\end{tabular}


Table V. Serum $\alpha$-MSH levels in SIRS group and non-SIRS group $(\mathrm{pg} / \mathrm{ml})$.

\begin{tabular}{lcc}
\hline Variable & No. & 1 day \\
\hline Non-SIRS group & 26 & $23.212 \pm 9.225$ \\
SIRS group & 22 & $13.334 \pm 4.931^{\text {a }}$ \\
t-test & - & 2.387 \\
P-value & - & 0.024 \\
\hline
\end{tabular}

${ }^{\mathrm{a} C}$ Compared with Non-SIRS group, $\mathrm{P}<0.05$.

the nervous system, $\alpha$-MSH can inhibit the activation of glial cells, thus inhibiting the production of TNF- $\alpha$, interleukin, NO and other factors (17). $\alpha$-MSH can also inhibit the local inflammatory response by inhibiting neutrophil chemotaxis $(15,16)$. In the event of an inflammatory reaction, the body produces anti-inflammatory factors such as IL-10. Catania found that $\alpha$-MSH can promote human monocyte IL-10 production and inhibit delayed allergic reactions (17). In addition, $\alpha$-MSH can interact with CNS signal transduction pathways to regulate the peripheral inflammatory response $(18,19)$.

Severe sepsis is usually accompanied by increased levels of inflammatory factors in the serum and a strong inflammatory response, while serum $\alpha$-MSH levels are reduced. During recovery from sepsis, serum $\alpha$-MSH levels increase, while serum $\alpha$-MSH levels remain low in patients without improvement, which may be associated with prognosis (20). In the nervous system injury, $\alpha$-MSH can promote axon growth (21), reduce apoptosis and reduce the damage caused by oxygenfree radicals and other substances. One study showed that the effect of $\alpha-\mathrm{MSH}$ on fever control was approximately 20,000 times of that of acetaminophen (22). The relationship between MSH and the central inflammatory response has been described in animal models in recent years. It was found that intraperitoneal injection of $\alpha$-MSH inhibited the activation of NF- $\mathrm{KB}$ in the brains of mice with LPS-induced central inflammatory responses. The results of that study also showed that $\alpha$-MSH could enter the CNS through the bloodbrain barrier, providing a theoretical basis for the usage of peripheral injection of $\alpha-\mathrm{MSH}$ substitutes in the treatment of central inflammation (22). In addition, serum $\alpha$-MSH content decreased in patients with craniocerebral trauma or subarachnoid hemorrhaging and sustained depression of serum $\alpha-\mathrm{MSH}$ levels was detrimental to patient condition (23). As a classic inflammatory factor, changes in serum TNF- $\alpha$ content reflect the degree of the inflammatory response. Therefore, TNF- $\alpha$ can be used as an indicator for the investigation of the correlation between the $\alpha$-MSH level and inflammatory response severity in patients with craniocerebral trauma. This result provides additional information for the better understanding of the association between $\alpha$-MSH and craniocerebral injury and its clinical significance. Results of the present study showed that serum $\alpha$-MSH levels in the trauma groups were lower than those in the healthy control group, possibly due to the consumption of serum $\alpha$-MSH after craniocerebral trauma. Serum $\alpha$-MSH levels were correlated with disease severity at $1,3,5$, and 7 days post-injury, and lower $\alpha$-MSH levels usually accompanied higher degrees of injury. Our study found a significant difference in the data between groups on the 7th day after injury, possibly due to variations in recovery extent caused by clinical infections and other complications. Serum $\alpha$-MSH levels first decreased, and then increased, with the lowest value found between 3 and 5 days after injury, which was consistent with the development of cerebral edema in clinical practice (24). The lowest $\alpha$-MSH levels in the severe group appeared earlier than that in other groups, further supporting that $\alpha-\mathrm{MSH}$ was reduced in the serum after injury. The more serious the disease, the more serum $\alpha$-MSH consumption occurred, which in turn further reduced serum content.

The present results showed that TNF- $\alpha$ levels in the serum were higher in the trauma groups than in the control group, and that serum TNF- $\alpha$ content was positively correlated with disease severity. Serum TNF- $\alpha$ levels in patients after craniocerebral trauma increased the first day after injury and peaked at the third day after injury, and then decreased gradually with patient recovery. Serum TNF- $\alpha$ levels in the severe group were significantly increased and peaked early after injury, but decreased at a much slower rate than the mild and moderate groups. The TNF- $\alpha$ level in the severe group at 5 and 7 days were still higher than at 1 day, indicating that more severe injury caused longer inflammatory responses. Serum $\alpha-M S H$ content was negatively correlated with TNF- $\alpha$ content at each time point. TNF- $\alpha$, as an inflammatory factor, can participate in inflammatory responses and reflect the degree of inflammation. As an endogenous anti-inflammatory peptide, $\alpha$-MSH content can be reduced by inflammation, possibly due to consumption by post-injury inflammation and limited production (24). Low levels of $\alpha$-MSH limit its antiinflammatory effect. The low levels of $\alpha-\mathrm{MSH}$ detected in the early stages after craniocerebral trauma indicated the presence of a strong inflammatory response and a high risk of SIRS. Our study found that serum $\alpha$-MSH levels at 1 day in patients with SIRS were significantly lower than in the non-SIRS group. Therefore, the detection of serum $\alpha$-MSH levels during the early stage after injury can be used to predict the risk of SIRS. Serum $\alpha$-MSH content in the patients of the survival group was higher than that of the death group, suggesting that continuously decreased $\alpha$-MSH levels reflected increased condition severity and poor prognosis. Further studies are required to test whether the supplementation of exogenous $\alpha$-MSH can effectively inhibit inflammation.

In conclusion, we believe that the serum $\alpha$-MSH levels during the early stage after craniocerebral trauma can be used to predict secondary SIRS, and sustained low levels of serum $\alpha$-MSH suggest poor prognosis.

\section{References}

1. Albrecht JS1, Hirshon JM, McCunn M, Bechtold KT, Rao V, Simoni-Wastila L, Smith GS: Increased rates of mild traumatic brain injury among older adults in US emergency departments, 2009-2010. J Head Trauma Rehabil 31: e1-e7, 2016.

2. Catania A, Gerloni V, Procaccia S, Airaghi L, Manfredi MG, Lomater C, Grossi L and Lipton JM: The anticytokine neuropeptide alpha-melanocyte-stimulating hormone in synovial fluid of patients with rheumatic diseases: Comparisons with other anticytokine molecules. Neuroimmunomodulation 1: 321-328, 1994. 
3. Jia L, Li GP, You C, Li H, Huang SQ, Yang CH, Xiong H and Zeng YJ: The epidemiology and clinical management of craniocerebral injury caused by the Sichuan earthquake. Neurol India 58: 85-89, 2010

4. Lin Y and Wen L: Inflammatory response following diffuse axonal injury. Int J Med Sci 10: 515-521, 2013.

5. Lu J, Goh SJ, Tng PY, Deng YY, Ling EA and Moochhala S: Systemic inflammatory response following acute traumatic brain injury. Front Biosci (Landmark Ed) 14: 3795-3813, 2009.

6. Mascarenhas L: Delayed posttraumatic acute subdural hematoma in elderly patients on anticoagulation. Neurosurgery 59: e1152, 2006.

7. Ichiyama T, Sakai T, Catania A, Barsh GS, Furukawa S and Lipton JM: Systemically administered alpha-melanocyte-stimulating peptides inhibit NF-kappaB activation in experimental brain inflammation. Brain Res 836: 31-37, 1999.

8. Muceniece R, Krigere L, Süli-Vargha H and Wikberg JE: Effects of alpha-melanotropin C-terminal tripeptide analogues on macrophage NO production. Peptides 24: 701-707, 2003.

9. Langlois JA, Rutland-Brown W and Wald MM: The epidemiology and impact of traumatic brain injury: A brief overview. J Head Trauma Rehabil 21: 375-378, 2006.

10. Hinson HE, Rowell S and Schreiber M: Clinical evidence of inflammation driving secondary brain injury: A systematic review. J Trauma Acute Care Surg 78: 184-191, 2015.

11. Witcher KG, Eiferman DS and Godbout JP: Priming the inflammatory pump of the CNS after traumatic brain injury. Trends Neurosci 38: 609-620, 2015.

12. Gyoneva S and Ransohoff RM: Inflammatory reaction after traumatic brain injury: Therapeutic potential of targeting cell-cell communication by chemokines. Trends Pharmacol Sci 36: 471-480, 2015.

13. Yousefzadeh-Chabok S, Dehnadi Moghaddam A, KazemnejadLeili E, Saneei Z, Hosseinpour M, Kouchakinejad-Eramsadati L, Razzaghi A and Mohtasham-Amiri Z: The relationship between serum levels of interleukins $6,8,10$ and clinical outcome in patients with severe traumatic brain injury. Arch Trauma Res 20: e18357, 2015.

14. Roberts I, Yates D, Sandercock P, Farrell B, Wasserberg J, Lomas G, Cottingham R, Svoboda P, Brayley N, Mazairac G, et al: Effect of intravenous corticosteroids on death within 14 days in 10,008 adults with clinically significant head injury (MRC CRASH trial): randomised placebo-controlled trial. Lancet 364:1321-1328, 2004.
15. Berberian V, Sánchez S, Sánchez-Borzone M, Attademo AM, Lasaga $\mathrm{M}$ and Celis ME: Effect of alpha-melanotropin hormone on serum levels of luteinizing hormone and progesterone in experimental rat autoimmune oophoritis. Peptides 27: 2295-2299, 2006

16. Catania A, Gatti S, Colombo G and Lipton JM: Targeting melanocortin receptors as a novel strategy to control inflammation. Pharmacol Rev 56: 1-29, 2004.

17. Catania A: Neuroprotective actions of melanocortins: A therapeutic opportunity. Trends Neurosci 31: 353-360, 2008.

18. Tracey KJ: Physiology and immunology of the cholinergic antiinflammatory pathway. J Clin Invest 117: 289-296, 2007

19. Macaluso A, McCoy D, Ceriani G, Watanabe T, Biltz J, Catania A and Lipton JM: Antiinflammatory influences of alpha-MSH molecules: Central neurogenic and peripheral actions. J Neurosci 14: 2377-2382, 1994.

20. Catania A, Cutuli M, Garofalo L, Airaghi L, Valenza F, Lipton JM and Gattinoni L: Plasma concentrations and anti-L-cytokine effects of alpha-melanocyte stimulating hormone in septic patients. Crit Care Med 28: 1403-1407, 2000.

21. Tanabe K, Gamo K, Aoki S, Wada K and Kiyama H: Melanocortin receptor 4 is induced in nerve-injured motor and sensory neurons of mouse. J Neurochem 101: 1145-1152, 2007.

22. Roth J, Zeisberger E, Vybíral S and Janský L: Endogenous antipyretics: Neuropeptides and glucocorticoids. Front Biosci 9: 816-826, 2004

23. Magnoni S, Stocchetti N, Colombo G, Carlin A, Colombo A, Lipton JM and Catania A: $\alpha$-melanocyte-stimulating hormone is decreased in plasma of patients with acute brain injury. J Neurotrauma 20: 251-260, 2003.

24. Jayakumar AR, Tong XY, Ruiz-Cordero R, Bregy A, Bethea JR, Bramlett HM and Norenberg MD: Activation of NF- $\kappa$ B mediates astrocyte swelling and brain edema in traumatic brain injury. J Neurotrauma 31: 1249-1257, 2014. 